\title{
Hardware Spiking Neural Networks: Slow Tasks Resilient Learning with Longer Term-Memory Bits
}

\author{
Pierre Lewden*, Adrien F. Vincent*, Charly Meyer*, Jean Tomas*, Shidoush Siami ${ }^{\dagger}$, and Sylvain Saïghi* \\ ${ }^{*}$ Laboratoire de l'Intégration du Matériau au Système, Univ. Bordeaux, Bordeaux INP, CNRS, Talence, France \\ Email: \{pierre.lewden, adrien.vincent, charly.meyer, jean.tomas, sylvain.saighi\}@ims-bordeaux.fr \\ $\dagger$ Communication and Networking Dept., IUT des Pays de l'Adour, Univ. Pau et des Pays de l'Adour, Pau, France \\ Email: ssiami@univ-pau.fr
}

\begin{abstract}
When aiming at efficient and low-power processing of event-based data, hardware implementations of spiking neural networks that co-integrate analog silicon neurons with memristive synaptic crossbar arrays are a promising framework. Fully analog systems however commonly make it difficult to learn patterns with real-world timescales, which are typically beyond the millisecond, due to intrinsic constraints of the underlying technologies. In this work, we propose to alleviate this issue by supplementing each presynaptic unit with a single memory bit, which allows to implement a hardware-friendly Spike TimingDependent Plasticity. By simulation means on the N-MNIST dataset, we illustrate the potential of this concept and show its robustness to postsynaptic neuron variability. We also discuss how to circumvent challenges raised by initial weight distribution. These results could facilitate the emergence of embedded smart systems directly fed by event-based sensors.

Index Terms-neuromorphic systems, spiking neural networks, memristors, spike timing-dependent plasticity
\end{abstract}

\section{INTRODUCTION}

Brain-inspired neuromorphic systems have attracted significant attention in the recent years [1], [2], offering better performance on cognitive memory-intensive tasks than conventional architectures limited by the von Neumann bottleneck [3]. In particular, leveraging Kirchhoff's laws and Ohm's law, neuromorphic architectures that rely on memristor-based synaptic arrays are candidates of choice to build highly integrated and low-power hardware implementations of Spiking Neural Networks (SNNs) with on-line learning capabilities. Among the possible learning rules, Spike Timing-Dependent Plasticity (STDP) naturally fits such hardware event-based systems and allows unsupervised learning, a major challenge for the processing of increasingly large volumes of data [4].

Implementing STDP in low-power analog or mixed-signal hardware however raises several challenges. First, depending on its complexity, the chosen STDP algorithm may not be hardware-friendly, inducing a consequential circuit or power overhead. Besides, the timescales that reasonably fit into integrated analog hardware are typically orders of magnitude shorter than the time constants of many real-time tasks.

Among the promising research works on hardware spiking neural networks that investigate fully CMOS solutions, some

Financial support from the French Agence Nationale de la Recherche (ANR) through MIRA project is acknowledged. This publication has received funding from the European Unions Horizon 2020 research innovation programme under grant agreement 732642 (ULPEC project). have decided to focus on time-accelerated tasks [5], which alleviates the need for implementing a slow paced learning rule. Subthreshold CMOS architectures may offer solutions to implement STDP-capable synapses that could handle reasonably long timescales, as suggested by the Dynamic Neuromorphic Asynchronous Processors (DYNAPs) architecture [6], at the expense of silicon area. Proof of concept of an experimental small-scale hardware architecture with $\mathrm{TiO}_{2}$ memristive synapses and on-line STDP-learning capabilities was recently reported by Prezioso et al. [7], with neurons that are however still implemented with discrete electronics and large capacitor values $(\sim 100 \mathrm{nF})$. Highly integrated and lowpower memristor-based hardware implementations of spiking neural networks with on-line learning capabilities still remain a challenging goal.

The following work derives from studies made to design a demonstrator of a smart vision sensor (European project ULPEC [8]) that co-integrates an event-based retinomorphic vision sensor [9] with a hardware spiking neural network implemented with analog CMOS neurons and a synaptic crossbar array of ferroelectric memristors. This paper makes several contributions in that context. First, we illustrate the need for a better match of the hardware time constants with the input event dynamics on a common event-based dataset $(\mathrm{N}$ MNIST [10]) and we introduce an accordingly modified STDP learning rule that requires little CMOS overhead (Section III). We then show the resilience of the system to two common variability issues of the postsynaptic circuits (Section IV). Finally, we study challenges posed by the initial synaptic weights on the learning and discuss two possible solutions (Section V).

\section{System ARchitecture AND Methodology}

In this work, we study the architecture shown in Fig. 1(a) using a Python in-house simulator that allows high-level description of the behavior of each hardware building block.

Each output Leaky Integrate-and-Fire (LIF) neuron includes a second generation current conveyor [11], which allows to apply postsynaptic voltage pulses onto the memristive synapses (Fig. 1(b-c)). To save power the current conveyor only transmits current if $i_{X}>0$. The output current $i_{Z}$ scales $i_{X}$ by a factor $K$ (constant in our simulations) in order to accommodate the limited value of the membrane capacitor 


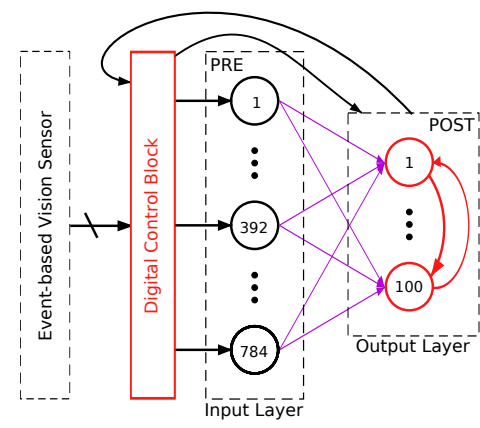

(a)

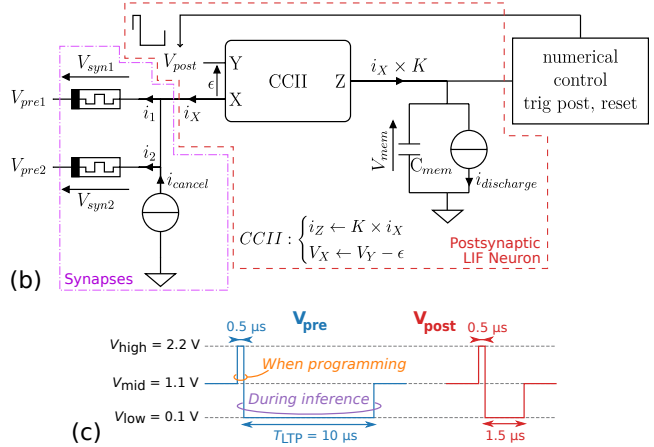

(c)

$T L T P=10 \mu \mathrm{s}$

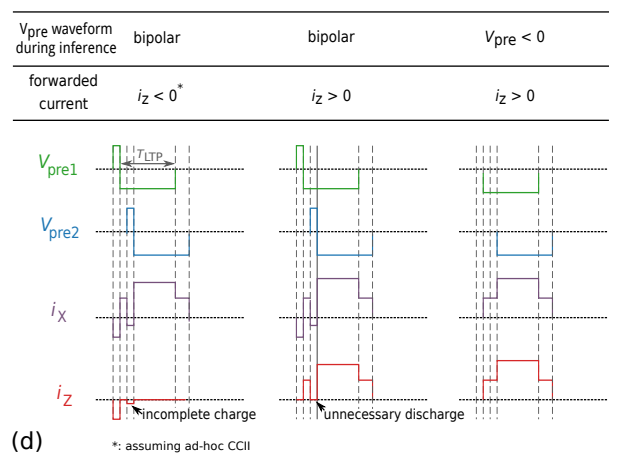

(d)

Fig. 1. Design overview. (a) System architecture: spiking neural network with $N_{\text {inputs }}=784$ and $N_{\text {outputs }}=100$ analog CMOS pre- and postsynaptic neurons (numbered circles) fully connected through a single layer of memristive synapses with no selection device (purple arrows). An event-based vision sensor feeds the network while a digital control block supervises the analog neuron operation. (b) Circuit-level overview of one Leaky Integrate-and-Fire (LIF) postsynaptic neuron, which includes a second generation current conveyor (CCII) block that only forwards positive currents (i.e., $\left.i_{X}>0\right)$. In this sketch, two input pixels are active and drive their respective memristor (devices on the left) while the rest of the memristive crossbar can be modeled as a current source $\left(i_{\text {cancel }}\right)$. For simplicity sake, we assume constant $V_{\mathrm{syn}}=-1 \mathrm{~V}$ for active synapses. The control block on the right is actually part of the digital control block in $(a)$. $(c)$ The pre- (left) and postsynaptic (right) voltage waveforms applied by the neurons. $(d)$ Comparing input voltage waveform strategies for inference and illustrating

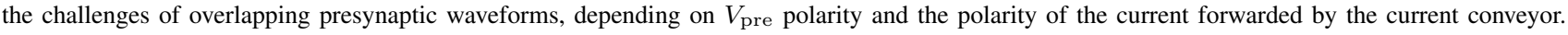

$C_{\text {mem }} \simeq 1 \mathrm{pF}$, while allowing to overcome the discharge $\left(\sim 100 \mathrm{~V} \cdot \mathrm{s}^{-1}\right)$ due to the constant leakage current $i_{\text {discharge }}=$ $100 \mathrm{pA}$ over the timescale of the targeted patterns. An output event is generated when the membrane voltage $V_{\text {mem }}$ reaches a threshold value ( $1 \mathrm{~V}$ in our simulations). Following a WinnerTake-All strategy, an output neuron that fires resets all the other output neurons. Besides, during training, a neuron that fired is reset until $N_{\text {refrac }}$ other output neurons have fired (in our simulations $N_{\text {refrac }}=10$ ), which prevents a single neuron from overtaking all the activity.

To prevent the shadowing of input events due to overlapping presynaptic pulses (Fig. 1(d)), we split the presynaptic pulse waveform into two components (Fig. 1(c)) that are applied independently. Inference pulses of duration $T_{\mathrm{LTP}}=10 \mu \mathrm{s}$ are triggered by events received from the input visual sensor and can participate to synaptic long term potentiation. Shorter $(0.5 \mu \mathrm{s})$ programming pulses can overlap with the lowamplitude part of a postsynaptic pulse for synaptic long term depression. From a circuit design viewpoint, the longer the time constants, the more chip area and energy is required.

If negative, the input voltage offset $\varepsilon$ of the current conveyor (Fig. 1(b)) continuously charges the membrane capacitor, resulting in spurious output events. Thus, one must ensure a positive offset value, e.g., by adding a dedicated low-resolution digital-to-analog converter used to decrease the voltage offset at the network setup.

Many memristive technologies can have their conductance tuned by applying a voltage pulse beyond a threshold value [12]. Among them, ferroelectric memristors offer extremely low values of conductance [13]. For simplicity sake and simulation speed, here we consider that the memristive devices are programmed accordingly to

$$
\Delta G=\left\{\begin{array}{ll}
+A_{\text {pot }} \times\left(G_{\max }-G_{0}\right) & \text { for } V_{\text {syn }} \leq-1.2 \mathrm{~V} \\
-A_{\mathrm{dep}} \times\left(G_{0}-G_{\mathrm{min}}\right) & \text { for } V_{\mathrm{syn}} \geq+1.2 \mathrm{~V} \\
0 & \text { otherwise }
\end{array},\right.
$$

where $G_{0}, G_{\min }$ and $G_{\max }$ are respectively the current, the minimal and the maximal conductance values, $V_{\mathrm{syn}}$ is the voltage applied onto the memristor (Fig. 1(b)), and $A_{\text {pot }}$ and $A_{\text {dep }}$ are (dimensionless) learning rates. Eq. (1) is reminiscent of a multiplicative Spike-Timing Dependent Plasticity (STDP) learning rule implemented with rectangular programming pulses of constant amplitude and duration. The precise characteristics of the STDP should have only a limited impact on the insights provided by our study as several works have shown the high resilience of similar architectures to synaptic variability or STDP curves [14], [15]. Based on recent work on ferroelectric devices [16], we consider $G_{\min }$ ranging from $1 \mathrm{nS}$ to $10 \mathrm{nS}$ and $G_{\max }$ from $0.1 \mu \mathrm{S}$ to $1 \mu \mathrm{S}$. We use $A_{\text {pot }}=A_{\text {dep }}$ $=0.1$, which allows single-epoch learning in our simulations.

\section{When One NeEds A Longer Term InPut Memory}

In all our simulations, we replace the event-based visual sensor with the N-MNIST dataset, a conversion of the MNIST handwritten digit pictures to an event-based framework using a neuromorphic vision sensor [10]. Each one of the 60,000 $(10,000)$ training (test) samples was generated through 3 consistent $100 \mathrm{~ms}$-saccades, resulting in 3 separate subclasses per digit class. Due to the limited amount of neurons in the hardware target, for each sample, we only use the data i) from the $28 \times 28$ central pixels ii) in the first saccade and iii) with ON polarity (i.e., increasing light events).

Fig. 2 illustrates a major issue of entirely implementing STDP through analog voltage overlapping. When an output neuron fires, because of the large mismatch between the analog hardware timescale $\left(\sim T_{\mathrm{LTP}}=10 \mu \mathrm{s}\right)$ and the input event rate ( $~ 7000$ events per second on average), only a handful of synapses are potentiated, and all the other ones are depressed. In our simulations, this leads to $\mathrm{a} \sim 0 \%$ recognition rate.

To overcome this issue-unspecific to N-MNIST-we propose an STDP variation by introducing a digital control block (Fig. 1(a)) that allows tracking input activity and driving the programming events. To keep the CMOS overhead as low as possible, we use a 1-bit flag per presynaptic neuron that is set to ON when the neuron receives an input event. When a postsynaptic neuron fires, the synapses that are connected 


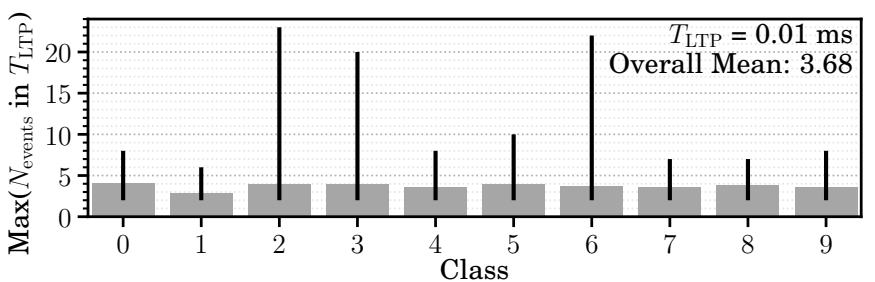

Fig. 2. For each digit class, statistics of the maximum amount of events $\left(N_{\text {events }}\right)$ that fall into a same long-term potentiation time window $\left(T_{\mathrm{LTP}}\right)$ over the first saccade of the 60,000 samples of the N-MNIST dataset. Gray large bars and black thin bars show the average value and the full range, respectively.

to it are then potentiated if their activity flag is $\mathrm{ON}$ and depressed otherwise. All presynaptic activity flags are then reset to OFF and we present the next digit sample. This form of STDP is thus entirely driven by postsynaptic events; each synapse connected to an output neuron that spikes is either potentiated or depressed, using separate pulses with constant duration and amplitude (translated into Eq. (1) first two lines in our simulations). This scheme is reminiscent of previous works in the literature [17], [18]. However, the time window that triggers long term potentiation is no more constant but depends on the last previous postsynaptic event. This makes the hardware implementation simpler: the timer needed for each presynaptic neuron is replaced with logic blocks.

Similarly to other works in the field, we focus on the subset of digits 5, 6 and 9 to reduce simulation time [19]. One epoch thus corresponds to 17,288 (2859) training (test) samples.

After training (for one epoch) and before testing, we disable output neurons that fired less than 10 times. For each remaining output neuron, if at least $60 \%$ of its last 10 events correspond to the same input class, we label it accordingly to the latter. Otherwise we disable the neuron. Empirically, this simple labeling strategy provides good agreement with labeling performed by a human, and the system achieves good selectivity (Fig. 3).

Without any device variability, the mean recognition rate is $92.4 \%$ (Fig. 4(a)), close to the $93.68 \%$ rate from the literature with a slightly different STDP and 400 output neurons [19]. (a)

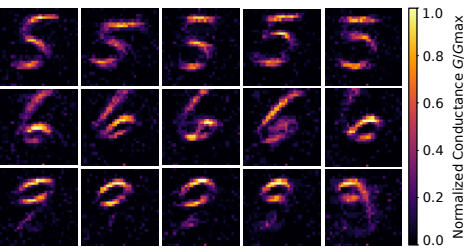

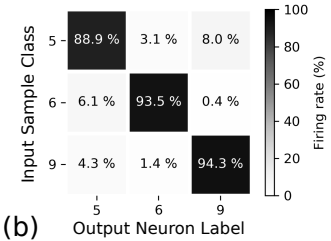

(b) Output Neuron Label
Fig. 3. Example of outcomes after one simulation run $\left(C_{\mathrm{mem}}=1 \mathrm{pF}\right.$, $\varepsilon=0 \mathrm{mV}, K=1 / 125$, initial weights uniformly distributed between $G_{\min }=10 \mathrm{nS}$ and $\left.G_{\max }=1 \mu \mathrm{S}\right)$. (a) Some conductance maps used after labeling. (b) Confusion matrix (on 2628 [218] correct [wrong] output events).

\section{IMPACT OF POSTSYNAPTIC NEURON VARIABILITY}

In a hardware implementation, some dispersion will affect the characteristics of the postsynaptic neurons, like the current conveyor offset voltages or the membrane capacitor values.

The wider the range of offset voltages $\varepsilon$, the larger the current $i_{\text {cancel }}$, which makes it harder for postsynaptic neurons

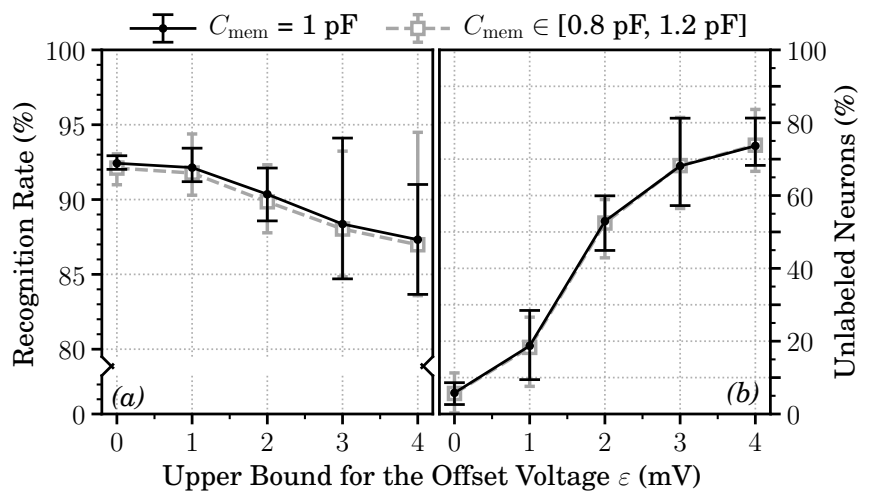

Fig. 4. Impact of the current conveyor offset voltage $(\varepsilon)$, without (black solid lines) and with uniformly distributed (gray dashed lines) dispersion of the postsynaptic neuron membrane capacitor values $\left(C_{\mathrm{mem}}\right)$. Random offset voltages $\varepsilon$ are uniformly drawn between $0 \mathrm{mV}$ and the upper bound reported along the x-axis. Results without (with) membrane capacitor dispersion are averaged over 25 (125) one-epoch runs, combining 5 random sets of initial conductance values with 5 random sets of offset voltages (and with 5 random sets of membrane capacitors). The random sets are kept consistent between the simulations as much as possible. The other system parameters are the same as in Fig. 3. Symbols show the average values and the error bars show the full ranges. (a) The recognition rate, and $(b)$ the fraction of output neurons (over $N_{\text {outputs }}=100$ neurons) that are not used for classification after labeling.

to spike. This results in significantly less active and selective output neurons after training. Cadence ${ }^{\circledR}$ Virtuoso Monte Carlo simulations of our current conveyor CMOS design suggest that the offset voltages $\varepsilon$ may mostly lie between $1 \mathrm{mV}$ and $2 \mathrm{mV}$. However, simulations of an even worse scenario (uniform distribution of $\varepsilon$ from $0 \mathrm{mV}$ to $4 \mathrm{mV}$ ) show that the recognition rate remains at $87.3 \%$ on average (solid black line in Fig. 4).

Besides, adding a challenging uniform dispersion of the membrane capacitor $C_{\text {mem }}$ between $\pm 20 \%$ around $1 \mathrm{pF}$ impacts only slightly more the performance (dashed gray line in Fig. 4). For example, the mean recognition rate drops to $86.9 \%$ with the largest offset voltage dispersion.

The results in this section suggest that the mixed-signal architecture we propose should remain operational even with levels of postsynaptic circuit variability that are plausible in a hardware implementation, provided that the redundancy among output neurons is sufficient (Fig. 4(b)).

\section{IMPACT OF INITIAL WeIght Distribution}

The mean conductance values of the patterns learned by the system determine a (rough) suitable range for postsynaptic parameters like the current conveyor factor $K$. Hence it is to be expected that the final system performance depends on the initial conductance distribution. This section illustrates how $<R_{\text {network }}>$, defined as the average resistance of the $N_{\text {inputs }}$ memristors connected to each output neuron, evolves in various situations and which challenges arise.

We typically scale the current conveyor factor $K$ respectively to the average of the expected final conductance maps $<G_{\text {final }}>_{\text {target }}$, in order to allow a reasonable triggering of the output neurons after a successful learning. Then, starting from a smaller $<R_{\text {network }}>$ (i.e., $<G_{\text {init }}>\geq<G_{\text {final }}>_{\text {target }}$ ) allows output neurons to fire and to learn (thick blue line in Fig. 5(b), with a $92.3 \%$ mean recognition rate after one 
epoch). Besides in such a situation Fig. 5(a) shows that a system with 10 times more resistive synapses (solid black line) behaves similarly to a less resistive learning-capable system (thick pink line), provided that $K$ is made 10 times stronger; in both cases, the mean recognition rate is $92.3 \%$. If on the contrary $<R_{\text {network }}>$ is initially too large (i.e., $<G_{\text {init }}>\ll$ $\left.<G_{\text {final }}\right\rangle_{\text {target }}$ ), the output neurons have difficulties to spike, which results in unsuccessful one-epoch learning (dashed red line in Fig. 5(b)).

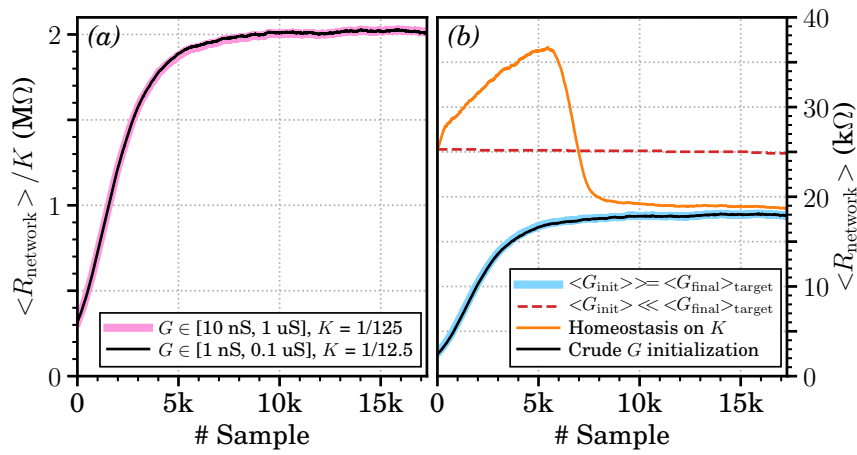

Fig. 5. Evolution of $\left\langle R_{\text {network }}\right\rangle$ the average resistance value seen by each output neuron. All results are averaged over five one-epoch simulation runs $\left(\varepsilon=0 \mathrm{mV}\right.$ and $\left.C_{\mathrm{mem}}=1 \mathrm{pF}\right) .(a)<R_{\text {network }}>$ normalized by the current conveyor factor $K$, when the synaptic crossbar is on average more resistive after the learning than initially. The thick pink line shows results with initial conductance values $G$ uniformly distributed between $10 \mathrm{nS}$ and $1 \mu \mathrm{S}$ and $K=1 / 125$ (average recognition rate [avgRR]: $92.3 \%$ ), while the thin black line corresponds to a case with ten times more resistive synapses and a factor $K$ scaled accordingly ( $K=1 / 12.5$, avgRR: $92.3 \%)$. (b) Illustration of challenges that arise when the synapses are initially more resistive on average than the patterns that would be learned after successful training. Conductance values can evolve between $G_{\min }=1 \mathrm{nS}$ and $G_{\max }=1 \mu \mathrm{S}$. If not mentioned, $K=1 / 125$. Solid thick blue line: a situation similar to $(a)$, for reference purposes (avgRR: $92.3 \%$ ). All three other cases use initial conductance values that are uniformly distributed between $G_{\min }$ and $G_{\max } / 10$. Dashed red line: unchanged learning strategy (avgRR: $0 \%$ ). Solid orange line: applying some homeostasis onto $K$ during the learning (starting from $K=1 / 12.5$, avgRR: $92.4 \%)$. Solid black line: half of the synapses are forcefully initialized to $G_{\max }$ before the learning (avgRR: $92.1 \%$ ).

A first approach to this challenge is to introduce an homeostasis process for each postsynaptic neuron, e.g., on $K$. For example, starting with conductance values similar to the previous failed attempt but with $K=1 / 12.5$ and decreasing it by 0.001 (down to $1 / 125$ ) each time the neuron spikes allows to reach a $92.4 \%$ mean recognition rate (solid orange line in Fig. 5(b)). Another solution is to explicitly initialize the synapses to decrease $<R_{\text {network }}>$. As an example, for each output neuron, when initially arbitrarily programming half of the synapses to their maximum conductance value $G_{\max }$, the system achieves $92.1 \%$ mean recognition rate (solid black line in Fig. 5(b)). Implementing the latter approach is likely to have a significantly smaller circuit-level overhead. Even the aforementioned crude memristor initialization allows to reach a good performance level; one could then simply leverage the programming blocks used for training to feed the digital control block with dedicated input events.

\section{CONCLUSION}

To mitigate the short learning timescale of analog CMOS, we proposed adding a binary memory to each presynaptic neuron. By simulation means, we illustrated the performance of our concept on the N-MNIST dataset and its resilience to neuron variability. We also studied how the initial synaptic weights impact the system operation, and how crude initialization or neuron homeostasis both mitigate this issue.

These results provide insights to build hardware spiking neural networks able to learn tasks with real-world timescales.

\section{REFERENCES}

[1] G. Indiveri and S. Liu, "Memory and information processing in neuromorphic systems," Proceedings of the IEEE, vol. 103, no. 8, 2015.

[2] C. S. Thakur, J. L. Molin, G. Cauwenberghs, G. Indiveri, K. Kumar, N. Qiao, J. Schemmel, R. Wang, E. Chicca, J. Olson Hasler, J.-s. Seo, S. Yu, Y. Cao, A. van Schaik, and R. Etienne-Cummings, "Large-scale neuromorphic spiking array processors: A quest to mimic the brain," Frontiers in Neuroscience, vol. 12, 2018.

[3] Editorial, "Big data needs a hardware revolution," Nature, vol. 554, no. 7691, 2018.

[4] Y. LeCun, Y. Bengio, and G. Hinton, "Deep learning," Nature, vol. 521, no. 7553, 2015.

[5] S. Friedmann, J. Schemmel, A. Grübl, A. Hartel, M. Hock, and K. Meier, "Demonstrating hybrid learning in a flexible neuromorphic hardware system," IEEE Transactions on Biomedical Circuits and Systems, vol. 11, no. $1,2017$.

[6] S. Moradi, N. Qiao, F. Stefanini, and G. Indiveri, "A scalable multicore architecture with heterogeneous memory structures for dynamic neuromorphic asynchronous processors (DYNAPs)," IEEE Transactions on Biomedical Circuits and Systems, vol. 12, no. 1, 2018.

[7] M. Prezioso, M. Mahmoodi, F. M. Bayat, H. Nili, H. Kim, A. F. Vincent, and D. B. Strukov, "Spike-timing-dependent plasticity learning of coincidence detection with passively integrated memristive circuits," Nature Communications, vol. 9, no. 1, 2018.

[8] Ultra-Low Power Event-Based Camera (ULPEC) H2020 project. [Online]. Available: http://ulpecproject.eu/

[9] C. Posch, T. Serrano-Gotarredona, B. Linares-Barranco, and T. Delbrück, "Retinomorphic event-based vision sensors: Bioinspired cameras with spiking output," Proceedings of the IEEE, vol. 102, no. 10, 2014.

[10] G. Orchard, A. Jayawant, G. K. Cohen, and N. Thakor, "Converting static image datasets to spiking neuromorphic datasets using saccades," Frontiers in Neuroscience, vol. 9, 2015.

[11] G. Lecerf, J. Tomas, S. Boyn, S. Girod, A. Mangalore, J. Grollier, and S. Saïghi, "Silicon neuron dedicated to memristive spiking neural networks," IEEE International Symposium on Circuits and Systems (ISCAS), 2014.

[12] D. Kuzum, S. Yu, and H.-S. P. Wong, "Synaptic electronics: materials, devices and applications," Nanotechnology, vol. 24, no. 38, 2013.

[13] A. Chanthbouala, V. Garcia, R. O. Cherifi, K. Bouzehouane, S. Fusil, X. Moya, S. Xavier, H. Yamada, C. Deranlot, N. D. Mathur, M. Bibes, A. Barthélémy, and J. Grollier, "A ferroelectric memristor," Nature Materials, vol. 11, no. 10, 2012.

[14] D. Querlioz, O. Bichler, P. Dollfus, and C. Gamrat, "Immunity to device variations in a spiking neural network with memristive nanodevices," IEEE Transactions on Nanotechnology, vol. 12, no. 3, 2013.

[15] T. Serrano-Gotarredona, T. Masquelier, T. Prodromakis, G. Indiveri, and B. Linares-Barranco, "STDP and STDP variations with memristors for spiking neuromorphic learning systems," Frontiers in Neuroscience, vol. 7, 2013.

[16] S. Boyn, J. Grollier, G. Lecerf, B. Xu, N. Locatelli, S. Fusil, S. Girod, C. Carrétéro, K. Garcia, S. Xavier, J. Tomas, L. Bellaiche, M. Bibes, A. Barthélémy, S. Saïghi, and V. Garcia, "Learning through ferroelectric domain dynamics in solid-state synapses," Nature Communications, vol. 8, 2017.

[17] B. Nessler, M. Pfeiffer, L. Buesing, and W. Maass, "Bayesian computation emerges in generic cortical microcircuits through spike-timingdependent plasticity," PLOS Computational Biology, vol. 9, no. 4, 2013.

[18] D. Querlioz, O. Bichler, A. F. Vincent, and C. Gamrat, "Bioinspired programming of memory devices for implementing an inference engine," Proceedings of the IEEE, vol. 103, no. 8, 2015.

[19] L. R. Iyer and A. Basu, "Unsupervised learning of event-based image recordings using spike-timing-dependent plasticity," International Joint Conference on Neural Networks (IJCNN), 2017. 